ORIGINAL ARTICLE

\title{
Intensified vmPFC surveillance over PTSS under perturbed microRNA-608/AChE interaction
}

T Lin ${ }^{1,2,9}$, A Simchovitz ${ }^{3,9}$, S Shenhar-Tsarfaty ${ }^{3,9}$, S Vaisvaser ${ }^{1}$, R Admon ${ }^{1}$, G Hanin ${ }^{3}$, M Hanan $^{3}$, E Kliper ${ }^{1}$, Y Bar-Haim ${ }^{2,4}$, N Shomron ${ }^{5}$,

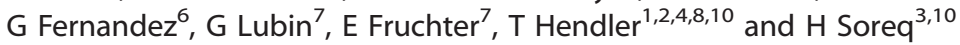

Trauma causes variable risk of posttraumatic stress symptoms (PTSS) owing to yet-unknown genome-neuronal interactions. Here, we report co-intensified amygdala and ventromedial prefrontal cortex (vmPFC) emotional responses that may overcome PTSS in individuals with the single-nucleotide polymorphism (SNP) rs17228616 in the acetylcholinesterase (AChE) gene. We have recently shown that in individuals with the minor rs 17228616 allele, this SNP interrupts AChE suppression by microRNA (miRNA)-608, leading to cortical elevation of brain AChE and reduced cortisol and the miRNA-608 target GABAergic modulator CDC42, all stressassociated. To examine whether this SNP has effects on PTSS and threat-related brain circuits, we exposed 76 healthy Israel Defense Forces soldiers who experienced chronic military stress to a functional magnetic resonance imaging task of emotional and neutral visual stimuli. Minor allele individuals predictably reacted to emotional stimuli by hyperactivated amygdala, a hallmark of PTSS and a predisposing factor of posttraumatic stress disorder (PTSD). Despite this, minor allele individuals showed no difference in PTSS levels. Mediation analyses indicated that the potentiated amygdala reactivity in minor allele soldiers promoted enhanced vmPFC recruitment that was associated with their limited PTSS. Furthermore, we found interrelated expression levels of several miRNA-608 targets including CD44, CDC42 and interleukin 6 in human amygdala samples $(N=7)$. Our findings suggest that miRNA-608/AChE interaction is involved in the threat circuitry and PTSS and support a model where greater vmPFC regulatory activity compensates for amygdala hyperactivation in minor allele individuals to neutralize their PTSS susceptibility.

Translational Psychiatry (2016) 6, e801; doi:10.1038/tp.2016.70; published online 3 May 2016

\section{INTRODUCTION}

Posttraumatic stress disorder (PTSD) is a severely debilitating psychiatric condition, especially in combat soldiers at high risk for trauma or chronic stress exposure. ${ }^{1,2}$ PTSD, however, develops only in a subset of those experiencing such events, ${ }^{3}$ pointing to individual differences in vulnerability and resiliency. The variable responses to stressful situations manifest in healthy stressexposed individuals as subthreshold posttraumatic stress symptoms (PTSS), known to increase the risk for PTSD ${ }^{4}$ and cause impairments in daily life situation, ${ }^{5,6}$ including difficulties in emotion regulation. ${ }^{7}$ Although several neural and genetic risk factors for PTSS are known, ${ }^{8-11}$ the molecular mechanisms underlying the variable intensity of such symptoms remain elusive.

Most imaging genetic studies in PTSS and PTSD focused on polymorphisms in coding regions of candidate genes. ${ }^{9,12,13}$ However, the great majority of genetic polymorphisms are located in noncoding regulatory regions. ${ }^{14}$ One of these is the singlenucleotide polymorphism (SNP rs17228616) C2098A substitution in the 'seed' domain of miRNA-608 binding site, at the 3'- untranslated region of the acetylcholinesterase ( $\mathrm{AChE}$ ) gene. We have recently shown that individuals with the minor A-allele demonstrate weakened interaction between miRNA-608 and the AChE gene. ${ }^{15}$ These individuals also exhibit reduced cortisol and their cortical tissues present excessive brain AChE hydrolytic activity, accompanied by decreases in other predicted neuronal protein targets of miRNA-608, including the Rho GTPase CDC42 involved in GABAergic signaling and the inflammation-regulating cytokine interleukin 6 (IL-6). These effects were attributed to weakened $\mathrm{AChE}$ interaction with the primate-specific regulatory miRNA-608, ${ }^{15,16}$ and are all stress-associated and predicting PTSS and PTSD. ${ }^{15,17,18}$ Intriguingly, a recent genome-wide association study has reported significant association between the rs78011900 SNP, which is in full-linkage disequilibrium with rs 17228616, and hypertension ${ }^{19}$ that is also related to PTSD. ${ }^{20}$ As the predicted cholinergic/GABAergic and inflammatory imbalance may modify the inherited risk or resiliency to develop stressrelated symptoms following stressful challenges, we initiated a study of the functional consequences of this genetic variation on the development of PTSS symptoms that indicate a risk of PTSD

\footnotetext{
${ }^{1}$ The Tel-Aviv Center for Brain Function, Wohl Institute for Advanced Imaging, Tel Aviv Sourasky Medical Center, Tel Aviv, Israel; ${ }^{2}$ School of Psychological Sciences, Tel Aviv University, Tel Aviv, Israel; ${ }^{3}$ The Edmond and Lily Safra Center for Brain Science, The Silberman Institute of Life Sciences, The Hebrew University of Jerusalem, Jerusalem, Israel; ${ }^{4}$ Sagol School of Neuroscience, Tel Aviv University, Tel Aviv, Israel; ${ }^{5}$ Department of Cell and Developmental Biology, Sackler Faculty of Medicine, Tel Aviv University, Tel Aviv, Israel; ${ }^{6}$ Donders Institute for Brain, Cognition and Behaviour, Department for Cognitive Neuroscience, Radboud University Medical Centre, Nijmegen, The Netherlands; ${ }^{7}$ Division of Mental Health, Medical Corps, Israel Defense Forces, Israel and ${ }^{8}$ Department of Physiology and Pharmacology, Sackler Faculty of Medicine, Tel Aviv University, Tel Aviv, Israel. Correspondence: Professor T Hendler, The Tel-Aviv Center for Brain Function, Wohl Institute for Advanced Imaging, Tel Aviv Sourasky Medical Center, 6 Weizmann Street, Tel Aviv 64239, Israel or Professor H Soreq, The Edmond and Lily Safra Center for Brain Science, The Silberman Institute of Life Sciences, The Hebrew University of Jerusalem, The Edmond J Safra Campus, 6 Weizmann Street, Jerusalem 91904, Israel.
}

E-mail: hendlert@gmail.com or hermona.soreq@mail.huji.ac.il

${ }^{9}$ These authors contributed equally to this work.

${ }^{10}$ Co-senior authors.

Received 9 September 2015; revised 14 March 2016; accepted 20 March 2016 
and the relation of the rs78011900 SNP to the threat neural circuitry.

The amygdala, hippocampus and prefrontal cortex have interrelated roles in PTSS and PTSD pathophysiology 8,21,22 and receive high levels of cholinergic and miRNA inputs. ${ }^{23,24}$ Rodent studies show that pharmacological and molecularly induced suppression of hippocampal AChE activity associate with increased anxiety- and depression-like behaviors and decreased resilience to repeated stress. ${ }^{25}$ This plausibly involves stressimpaired hippocampal inhibitory feedback to the amygdala, ${ }^{26}$ which may lead to enhanced amygdala reactivity, a hallmark and a predisposing factor of PTSD. ${ }^{8,24,27-29}$ Excessive acetylcholine (ACh) release in the amygdala strengthens the associations between environmental stimuli and stressful events, potentially contributing to maladaptive learning and underlying affective disorders. ${ }^{30}$ Human studies indeed demonstrate that increases in $\mathrm{ACh}$ signaling contribute to stress-related illnesses, such as major depression. ${ }^{31}$ As the $r$ 17228616 polymorphism is related to excessive brain AChE hydrolytic activity, ${ }^{15}$ we surmised that the AChE gene is close to its maximal expression capacity in individuals with the minor allele. This would limit their ability to react to a changing environment by overproducing $\mathrm{AChE}$ to suppress the induced stress. We expected that this would be manifested by enhanced neural response of the threat circuit, including the amygdala which is rich in both GABAergic synapses and AChE. ${ }^{32}$ In addition, animal studies have demonstrated involvement of inherited tendencies of $\mathrm{AChE}$ increases in response to stress, $^{33}$ miRNA suppression of brain AChE (by miRNA-132) which limits stress-induced cognitive impairments, ${ }^{34}$ and CDC42 suppression leading to anxiogenic reaction. ${ }^{15}$ Therefore, we predicted modulated PTSS reactions in individuals with the minor allele of the $r$ s 17228616 SNP.

How individuals compensate for risk-associated genotypes is a challenging, scarcely studied issue. A neural candidate for coping with exaggerated amygdala activity is the ventromedial prefrontal cortex (VmPFC). This region is activated when a negative emotional response is suppressed, in a manner related to the elimination of previously learned associations of threatconditioned signals. ${ }^{35,36}$ Under normal conditions, suppressing messages by the vmPFC lead to amygdala downregulation in response to negative stimuli, whereas PTSD patients show less activity in the vmPFC than healthy volunteers when experiencing or regulating emotions. ${ }^{37}$ This indicates vmPFC involvement in the process of regulation and thus resilience to trauma. In the current study, we used functional magnetic resonance imaging (fMRI) to examine how the rs17228616 polymorphism in the AChE gene impacts PTSS following chronic military stress exposure; specifically, we asked whether it serves as a vulnerability, resilience or an inert factor, and what are its functional influences on the human amygdala and vmPFC responses to stressful challenges.

\section{MATERIALS AND METHODS}

\section{fMRI experiment}

Participants. The participants were 76 Israel Defense Forces soldiers (13 females; age 18-19 years) who experienced 1 year of chronic military stress during their mandatory service. Of those, 29 participants (Group A) served as combat paramedics and 47 (Group B) were recruited after 1 year of intensive and advanced combat training. During military training, the participants were exposed to a wide variety of stressful physical and psychological demands, including sleep restrictions, prolonged periods of physical survival challenges, face-to-face combat training and counterterrorism combat course, that have been shown to affect well-being ${ }^{38}$ and may impact the development of stress-related symptoms. ${ }^{1,2}$ The fMRI data of Group A was already published by our group. ${ }^{29}$ As there were no differences in amygdala and VmPFC activity or in PTSS between paramedics and soldiers in training (all $F<1.28, P>0.25$ ), all 76 volunteers were considered as a single group. All participants had no reported history of psychiatric or neurological disorders, no current use of psychoactive
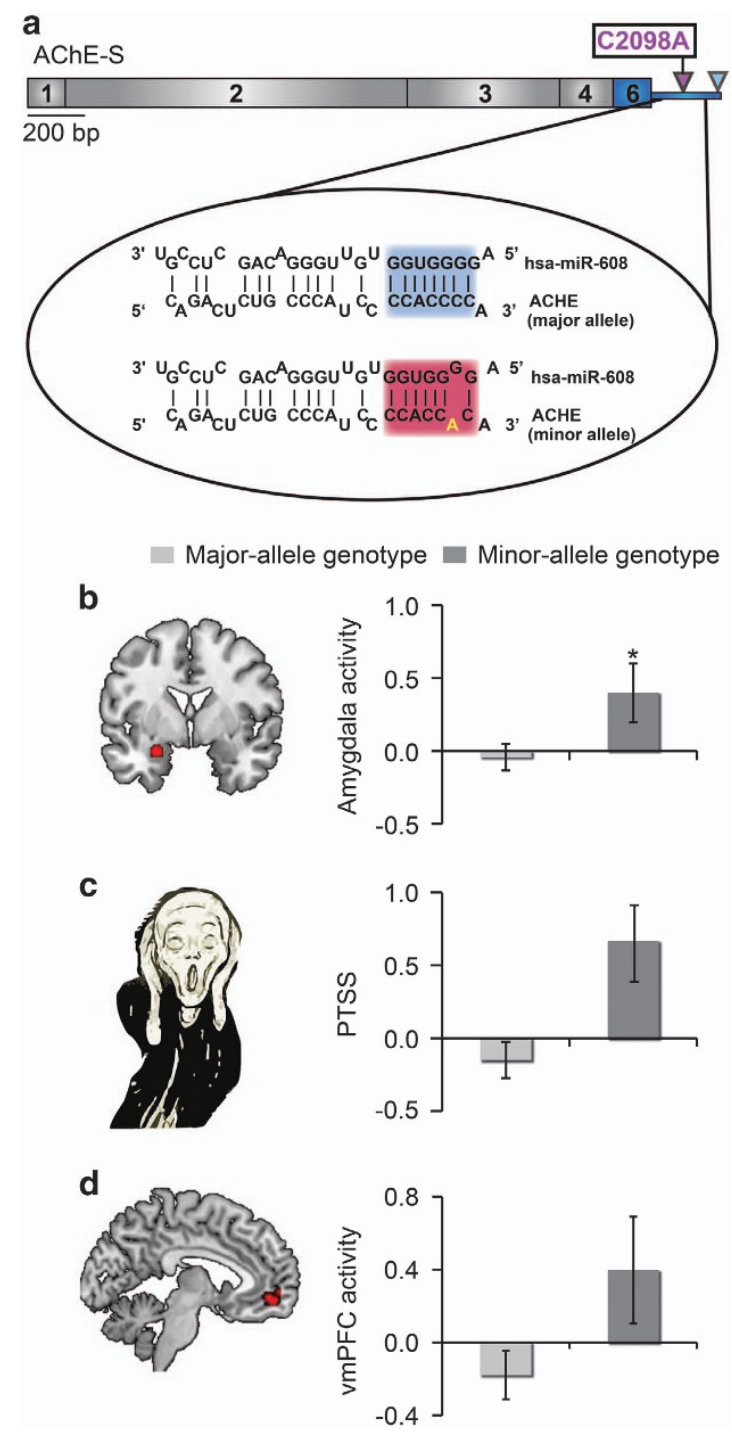

Figure 1. rs17228616 single-nucleotide polymorphism impairs hsamiR-608/AChE interaction. (a) Schematic illustration of the AChE transcript, exon numbers and the rs17228616 location, with the $C$ to A (C2098A) major to minor allele substitution interrupting hsamiR-608 binding. (b) Increased activation within the amygdala region of interest ( $\mathrm{ROI} ;-28,0,-21)$ for the minor allele $(A A, A C$, $n=13$ ) relative to the major allele (CC, $n=63$ ) group. (c) Limited elevation in posttraumatic stress symptoms (PTSS, z-score) in the minor allele (AA, AC, $n=13$ ) relative to major allele $(C C, n=58)$ group. (d) Increased activation within the ventromedial prefrontal cortex (vmPFC) ROI $(0,50,-12)$ for the minor allele (AA, AC, $n=13$ ) relative to the major allele $(C C, n=63)$ group. The data are represented as mean \pm s.e. Bar charts in $\mathbf{a}$ and $\mathbf{c}$ show the mean of beta values across all voxels in the ROls for emotional versus neutral stimuli. ${ }^{*} P<0.05$. AChE, acetylcholinesterase.

drugs, no family history of major psychiatric disorders and no incidence of childhood abuse or potentially traumatic events before military enrollment.

Genotyping. DNA was extracted from saliva samples collected with an OrageneTM DNA Self-Collection Kit in Group A and from blood samples in group B, using commercial kits. Genotyping of the A-allele of $r$ s 17228616 (C2098A) versus the C-allele (Figure 1a) was performed using TaqMan genotyping primers and AccuStart genotyping ToughMix low ROX (Quanta BioSciences, Gaithersburg, MD, USA). To further differentiate between homozygous (AA) and heterozygous (CA) rs17228616 individuals, PCRamplified DNA was fully sequenced. The participants were categorized 
according to DNA sequencing: carriers of one or two copies of the minor A-allele of $r$ 17228616C (AA/CA) were assigned to the minor allele group $(N=13)$, whereas $C$ homozygotes (CC) were assigned to the major allele group $(N=63)$.

fMRI visual task. The participants viewed backward-masked colored photographed pictures of three contents (military, medical and neutral) presented for either 33 or $83 \mathrm{~ms}$ in a block design and were asked to indicate whether they recognized a person or an object as described in ref. 29 (see Supplementary Materials and Methods for details).

Assessment of PTSS. The PTSS levels in Group A were evaluated using the posttraumatic stress diagnostic scale questionnaire (PDS). ${ }^{39}$ In Group B, the symptoms were evaluated with the military version of the 17-item PTSD checklist, $^{40}$ which specifically asks about symptoms related to stressful military experiences. As each of these questionnaires uses different scales, we calculated $z$-scores for each group, enabling to integrate data from both groups. Five participants did not complete the PTSS questionnaires, and thus were excluded from analyses concerning this variable.

Mediation analysis. The mediation indirect effects were tested using the PROCESS macro in SPSS, ${ }^{41}$ which tests indirect effects using a bootstrap approach. The indirect effect was considered to be significant if its $95 \%$ bootstrap confidence intervals from 10000 iterations did not include zero at $P=0.05$. In all indirect effect analyses, A-allele carriers were coded as 1 .

\section{Amygdala brain samples}

The dissected amygdala tissues from non-demented control volunteers $(n=7$, three males, four females) were provided by the Netherlands Brain Bank (Supplementary Table S1). Written informed consent from donors or their next of kin, as well as ethical approval was obtained in all the cases. The tissues were kept at -70 degrees until RNA extraction, and were used for quantitative PCR analysis. RNA extraction from brain tissues, CDNA synthesis, quantitative PCR measurements and data analysis were essentially performed as in refs 15,16 (see Supplementary Materials and Methods for details). DNA was extracted as described in ref. 15 followed by genotyping of $\mathrm{PCR}$ product amplification via Illumina sequencing. Unfortunately, we could not find human vmFPC tissues, so we could not extend these tests to the vmPFC.

\section{RESULTS}

The coding regions of the genome account for less than $2 \%$ of the entire human DNA, ${ }^{42}$ and numerous noncoding genomic domains contribute to individual differences in reactivity to neural diseases, including threat and its regulation. ${ }^{43,44}$ Nevertheless, most anxiety-related imaging genetic studies focus on those simpleto-explain polymorphisms in the coding regions of candidate genes. ${ }^{9}$ In the present study, we selected the $r$ s 17228616 SNP in the noncoding region of the AChE gene to investigate geneenvironment brain interactions as those are reflected in PTSS. This was achieved by combining genotype and brain transcript tests with fMRI measurements of amygdala and vmPFC responses to emotional stimuli in a military population at high risk for chronic stress exposure, known to impact the development of stressrelated symptoms. ${ }^{1,29}$

\section{Genotyping results}

The rs 17228616 SNP in the AChE gene was present in $17 \%$ of 76 participants, fourfold higher incidence than in Caucasians but twofold less than in African American. ${ }^{15}$ Thirteen participants (17.1\%) formed the minor A-allele group (AA, AC) and 63 participants $(82.9 \%)$ with the CC genotype formed the major allele group (Figure 1a). This distribution accords with another study from our group identifying the minor allele variant in $17 \%$ of over 900 tested Israeli participants (Shenhar Tsarfaty et al., in preparation). The ethnic differences in the prevalence of this SNP are compatible with evolution studies of miRNAs in population migrations ${ }^{45}$ that should be considered in functional studies of the brain-related implications of this and other polymorphisms.
Genotype effects on limbic responses and PTSS following emotional stimuli

The amygdala and hippocampus are rich in both GABAergic synapses ${ }^{46}$ and $\mathrm{AChE}^{32,47}$ suggesting that their activity may be modulated by the cholinergic/GABAergic imbalance imposed by the rs 17228616 polymorphism in the AChE gene. Compatible with this prediction, genotypes with the minor allele $(A C, A A)$ were associated with greater amygdala reactivity to emotional stimuli compared with other volunteers (Mann-Whitney $U=269.0$, $P<0.05$; Figure 1b). In contrast, the rs17228616 SNP did not influence hippocampus function (Mann-Whitney $U=308.0$, $P=0.16$ ). Moreover, despite their neurogenetic vulnerability, ${ }^{8,15}$ PTSS levels did not differ between minor allele and major allele individuals (Mann-Whitney $U=267.0, P=0.09$; Figure 1c). This accords with recent genome-wide association studies, concluding that the genetic architecture of PTSD may be determined by many SNPs with small effects that are not directly related to the cholinergic pathway. ${ }^{48,49}$ For the AChE gene, this outcome plausibly reflects the exceedingly high G, C content that complicates genome-wide association study data collection. It is also possible that, in this study, the small sample size and low variability in PTSS hindered the detection of a genotype-PTSS association.

Genotype effects on prefrontal responses to emotional stimuli The great majority of stressed individuals cope with their inherited genome features and do not develop excessive PTSS or PTSD. To explore a possible route that enables minor allele individuals to compensate for the imbalanced amygdala signaling, we compared the reactions of their VMPFC, a key component of the cortico-limbic circuit involved in the top-down regulation of affective subcortical circuitry, to those of their major allele peers. The rs17228616 minor allele group members showed marginally significant threefold higher vmPFC activation than the major allele group to emotional versus neutral stimuli (Mann-Whitney $U=272.0, P=0.058$; Figure $1 \mathrm{~d}$ ). Although this finding is only marginally significant, we speculated that it might indicate that greater vmPFC functioning may operate as a clinically helpful compensatory mechanism for A-allele individuals to neutralize their sensitivity to stress, and that they present no increase in PTSS owing to the recruitment of the vmPFC to overcome their stress vulnerability.

\section{Relationship between genotype, brain response and PTSS}

To test the above-mentioned theory in the search for potential functional relevance of the neural patterns that might explain the limited difference in PTSS between minor and major allele individuals, we conducted an indirect effect analysis using a bootstrapping approach. ${ }^{50}$ This method avoids the power problem introduced by non-normality and is less restricted by sample size. This mediation analysis revealed that volunteers with the minor allele exhibited exacerbated amygdala reactivity to emotional stimuli, which in turn was associated with increased vmPFC activity (indirect effect $=0.36 *, 95 \%$ confidence interval: 0.04 to 0.83 ; Figure 2a). The observed pattern of vmPFC hyperactivity accompanied by increased amygdala activity as found in the minor allele group is strikingly different from previous reports of hyperresponsive amygdala accompanied by hyporesponsive vmPFC in PTSD, ${ }^{51}$ suggesting that minor allele individuals may possibly achieve limited PTSS by over-recruiting the vmPFC. To verify this conclusion, a second mediation model was tested, which yielded a significant indirect effect of genotype on PTSD symptoms via vmPFC activation (indirect effect $=-0.14^{*}$, $95 \%$ confidence interval: -0.44 to -0.007 ; Figure $2 b$ ), such that the greater vmPFC response to emotional stimuli in volunteers with the A-allele was associated with lower levels of PTSS 

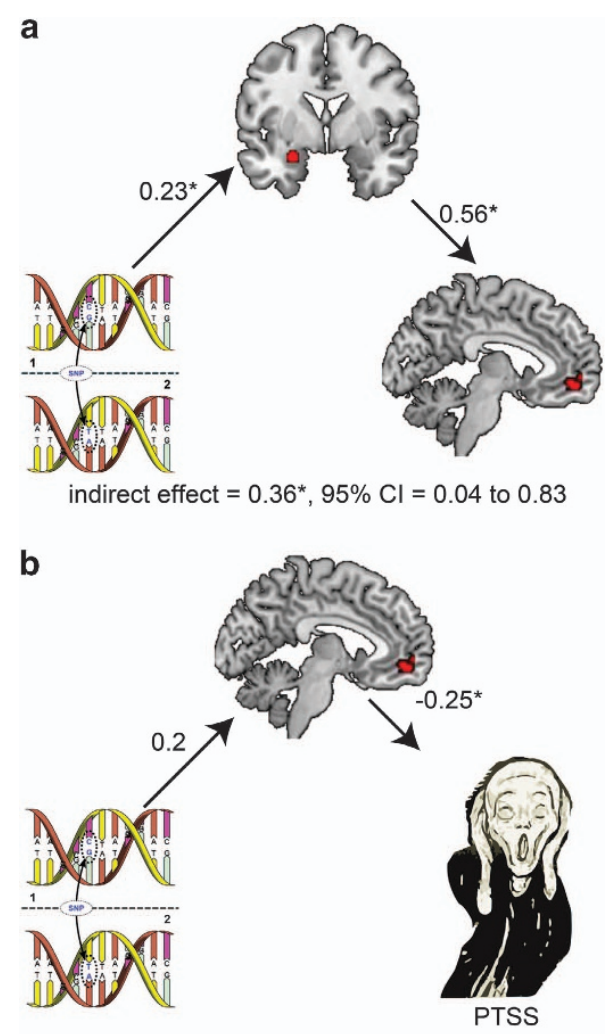

indirect effect $=-0.14^{*}, 95 \% \mathrm{Cl}=-0.44$ to -0.007

Figure 2. Models of the relationship between $r$ 17228616 genotype, brain activity and posttraumatic stress symptom (PTSS). (a) The illustrated mediation model depicts a significant indirect path of the effect of genotype on ventromedial prefrontal cortex (vmPFC) activity via amygdala activation. Specifically, A-allele carriers exhibited greater amygdala reactivity to emotional stimuli, which in turn was associated with increased vmPFC activity. (b) The illustrated mediation model depicts a significant indirect effect of genotype on PTSS via vmPFC activation. Specifically, A-allele carriers exhibited greater vmPFC response to emotional stimuli, which in turn was associated with lower levels of PTSS symptoms. The $\beta$ values are shown next to the arrows indicating each link in the analysis. ${ }^{*} P<0.05 .95 \% \mathrm{Cl}, 95 \%$ confidence interval.

compared with major allele carriers. Conversely, the indirect effect of genotype through the amygdala was insignificant (indirect effect $=-0.09,95 \%$ confidence interval: -0.39 to 0.03 ), compatible with the minor allele group's limited increase of trait anxiety ${ }^{15}$ and PTSD symptoms (Figure 1b). Although mediation analyses should be interpreted with caution and replications of these results are required to draw firm conclusions, we conclude that the rs17228616 polymorphism exacerbates amygdala activity, but is not a PTSS risk factor as it promotes vmPFC hyperactivation, which overcomes the exaggerated amygdala response.

Expression of the primate-specific miRNA-608 and its targets in the human amygdala

Animal studies demonstrate that miRNAs expressed in the amygdala may be involved in the stress response, ${ }^{52}$ but previous tests of miRNA-608 and its targets in the human brain were limited to the temporal cortex. ${ }^{15}$ To assess expression of miRNA-608 and its target genes in the amygdala, we performed quantitative PCR with reverse transcription measurements from amygdala tissues of healthy human adults received from the Netherlands Brain Bank. These analyses confirmed expression, albeit at a modest level of miRNA-608 in the amygdala of seven donors, all homozygotes for the major allele of the rs17228616 SNP a
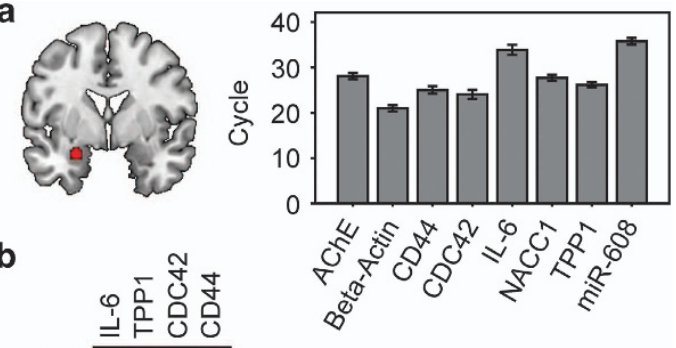

b
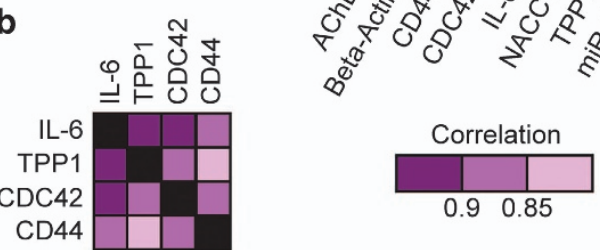

Figure 3. Interrelated expression of miR-608 and its targets in the amygdala. (a) Human amygdala (marked red) served to extract RNA. hsa-miR-608, as well as six of its verified and predicted targets, including acetylcholinesterase (AChE) and CDC42 and the normalizing beta-actin gene are all expressed in the human amygdala (quantitative PCR (qPCR) cycle $<34$ for targets, qPCR cycle $<36$ for miRNA-608). Shown are Cq values as measured by qPCR. (b) Several pairs of verified and predicted targets of hsa-miR-608 show positively correlated expression patterns, normalized to beta-actin and RIN (RNA integrity number) values $(P<0.05$, false discovery rate corrected). Color code shows correlation matrix intensity. IL-6, interleukin 6; miRNA, micro RNA.

(Figure 3a). We also observed expression of several miRNA-608 targets, including AChE in these tissues (Figure 3a). Moreover, our measurements revealed positive correlations between several pairs of both predicted and verified brain-expressed miRNA-608 target transcripts, following RNA quality and beta-actin normalization. For the seven brains, we observed significant positive correlations between the GABAergic regulating $\mathrm{CDC42}$, the immune biomarker CD44, the pro-inflammatory cytokine IL-6 and the telomere-related TPP1 transcript (all correlations passed false discovery rate correction of 0.05 , see Table 1 and Figure $3 \mathrm{~b}$ ). The fact that these transcripts are all expressed in the amygdala is essential for and compatible with the proposed mechanism of action; and the correlated expression levels indicated common regulatory mechanism(s) for these transcripts in the human amygdala, which could include miRNA-608-mediated control. These findings further predicted that higher brain AChE levels, as shown for individuals with the minor allele, would be associated with reduced and interrelated levels of CDC42, CD44 and IL-6. This can imbalance cholinergic and GABAergic signaling, and promote inflammation and consequent anxiety ${ }^{15}$ (Figure $3 \mathrm{~b}$ ). There was no apparent effect of age or gender.

\section{DISCUSSION}

The rs17228616 SNP interferes with AChE suppression by the cholinergic controller and primate-specific miRNA-608 ('CholinomiR', $\left.{ }^{53}\right)$, and its occurrence in healthy adults is associated

Table 1. Correlations between brain-expressed miRNA-608 target transcripts

\begin{tabular}{lcccc}
\hline & $C D 44$ & $C D C 42$ & $I L-6$ & $T P P 1$ \\
\hline CD44 & 1 & $0.901(0.028)$ & $0.983(0.002)$ & $0.900(0.028)$ \\
CDC42 & $0.901(0.028)$ & 1 & $0.861(0.033)$ & $0.849(0.033)$ \\
IL-6 & $0.983(0.002)$ & $0.861(0.033)$ & 1 & $0.865(0.033)$ \\
TPP1 & $0.900(0.028)$ & $0.849(0.033)$ & $0.865(0.033)$ & 1 \\
\hline
\end{tabular}

Abbreviations: IL-6, interleukin 6; miRNA, micro RNA. Significant correlations between pairs of transcripts are shown along with the respective post-false discovery rate $P$-values (in parentheses). 
SNP-induced changes

Stress-induced changes

Stress and SNP interaction

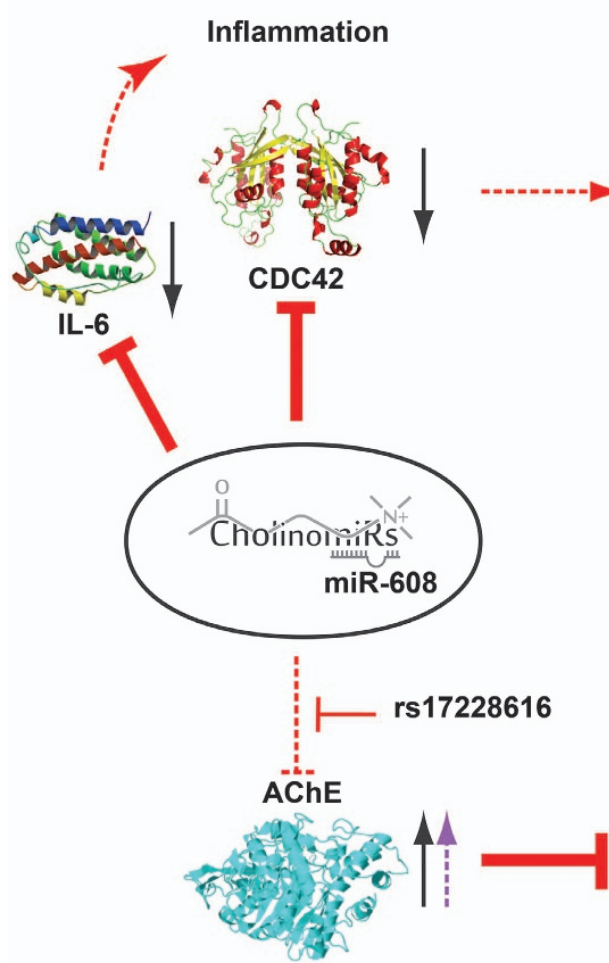

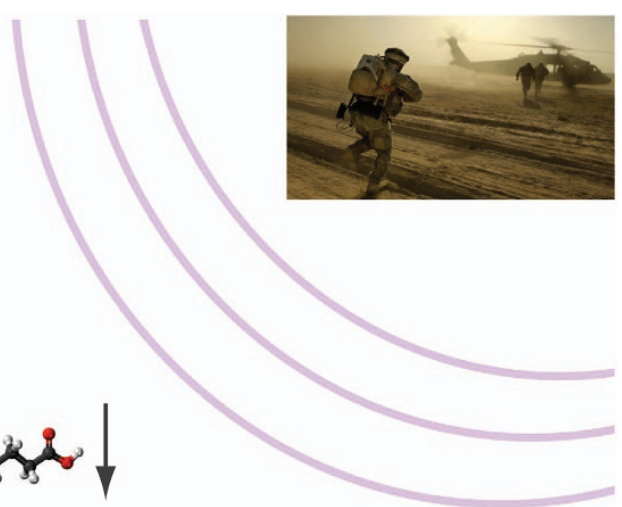

GABA
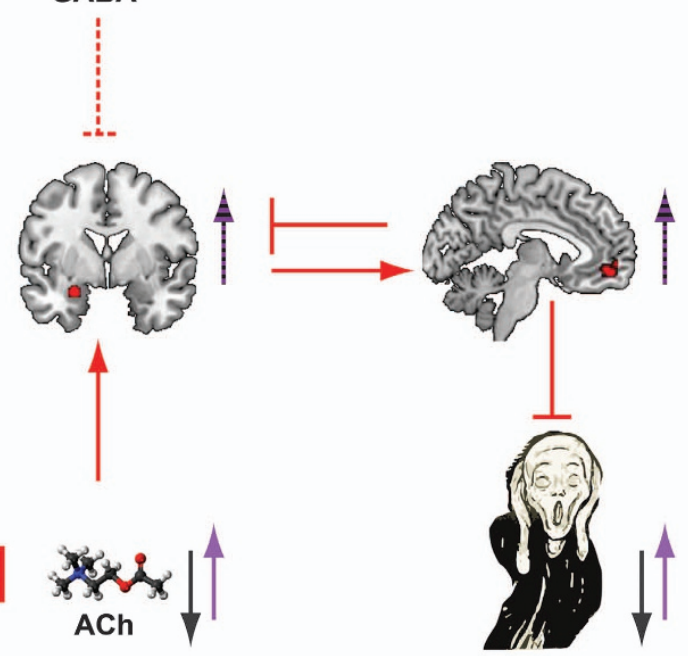

Stress-related symptoms

Figure 4. Schematic presentation of how the rs17228616 single-nucleotide polymorphism (SNP) in the acetylcholinesterase (AChE) gene may affect the vulnerability to stress. This SNP interrupts miRNA-608/AChE interaction, leading to (1) elevated brain AChE activity and thus reduced cholinergic signaling, (2) reduction in the GABAergic modulator CDC42, limiting the inhibitory GABAergic signaling and (3) reduced IL-6 levels, effecting inflammatory pathways. Together, these signaling pathway impairments may enhance amygdala reactivity to emotional stimuli of minor allele individuals due to insufficient suppression of stress-related increases in cholinergic signaling and reduced inhibitory GABAergic signaling. As a coping mechanism under trauma load, these individuals over-recruit the ventromedial prefrontal cortex to downregulate the amygdala, and thus develop limited posttraumatic stress syndrome. Broken arrows represent reduced inhibitory input. The effects of this SNP, of stress exposure and of SNP-exposure interactions are represented by black, purple and striped black/purple arrows, respectively. IL-6, interleukin 6; miRNA, micro RNA.

with multiple anxiety- and inflammation-related changes: elevated brain AChE activity and correspondingly reduced brain levels of the GABAergic-related Rho GTPase CDC42, suppression of which induces anxiety, and the inflammation-regulating cytokine IL-6. ${ }^{15}$ MiRNA-608 has recently been identified as a tumor-suppressive miRNA, ${ }^{54,55}$ and has been proposed as a potential novel therapeutic target for treating osteoarthritis. ${ }^{56}$ Yet, because miRNA-608 is primate-specific, ${ }^{45}$ previous animal experiments of miRNA/AChE interactions and stress ${ }^{34}$ cannot be reproduced here to determine a causal link to stress. Despite this difficulty, the current study points to the involvement of miRNA-608 in stressrelated neural reactions. We demonstrated that following chronic stress, individuals with the rs17228616 minor allele enhance neural activity in the amygdala accompanied by non-pathological PTSS levels. This finding was supplemented with a marginal increase in vmPFC response. Our model predicts multileveled effects of this polymorphism on the neural response to emotional stimuli within the threat circuit and its association with PTSS, as is schematically summarized in Figure 4. We further found expression of miRNA-608 and several of its verified and predicted target transcripts in the amygdala, including AChE, CDC42, CD44, NACC1 and IL-6; A subset of these, involved in inflammation, showed very tight positive interrelated correlations (CDC42, CD44, IL-6 and TPP1), indicating common regulation by miR-608, among other potential causes. Our previous work has shown such correlation in the temporal gyrus, expanding the implications of our current analyses beyond the amygdala. To the best of our knowledge, our current study is the first to implicate a SNP in a noncoding region in measurable brain fMRI changes under emotional stimuli, and the first to suggest a functional coping mechanism with a riskassociated SNP.

Although PTSS were not directly related to the polymorphism, our fMRI results provide support for the relation between the rs17228616 polymorphism and PTSS by demonstrating that individuals with the rs17228616 minor allele show heightened amygdala response to emotional stimuli. This is compatible with earlier functional neuroimaging studies reporting exaggerated amygdala activation in patients with PTSD compared with control subjects $^{27,28}$ and support the notion that amygdala hyperactivation is a predisposing factor for developing stress-related disorders. ${ }^{8,29}$ The finding that minor allele individuals show 
hyperactive amygdala but do not present elevated PTSS has led us to jointly investigate how they cope with stressful challenges.

The rs 17228616 minor allele group showed a trend of increased vmPFC activation compared with the major allele group. This finding was further strengthened by a significant indirect path analysis showing that soldiers with the minor A-allele also exhibited higher levels of amygdala activity compared with those with the major C-allele, and that the minor allele was involved in increased recruitment of the vmPFC in these volunteers. The results of this mediation analysis suggest that minor allele individuals may recruit the VmPFC to downregulate their elevated amygdala reactivity more effectively than the major allele group. An additional mediation analysis suggested that the elevated vmPFC activity has a clinically protective role in A-allele volunteers, such that they attained lower than predicted levels of PTSS compared to major allele carriers by increasing the recruitment of their VmPFC. However, other, yet-unknown explanations for these fMRI results may exist. To validate or exclude this hypothesis, future studies could test whether parallel vmPFC hyperactivation occurs in other cases where the inherited tendency to develop PTSS is compensated for, and whether PTSD patients with the A-allele lack this compensatory mechanism and thus develop and maintain high levels of symptoms.

Amygdala enhancement in minor allele individuals may be driven by either the imbalanced cholinergic, GABAergic and/or inflammation signaling, which are all intensified under stressful environments ${ }^{57}$ (Figure 4). Apart from their direct effects on the amygdala, GABA and acetylcholine could possibly influence this phenotype indirectly through other regions within the threat circuit, such as the hippocampus. ${ }^{24,58,59}$ However, the $r s 17228616$ SNP was not associated with modified hippocampus activity, suggesting that individual differences in the AChE system likely explain only part of the neural changes associated with PTSS. This finding also excludes hippocampal involvement in the limited PTSS of minor allele individuals. An alternative explanation is that although their reduced cholinergic signaling causes decreased hippocampal activity, the hippocampus is upregulated back to normal levels by the overrecruitment of the vmPFC. ${ }^{35}$ To elucidate this, focused studies of the effects of this gene on hippocampusvmPFC connectivity are needed.

The noncoding SNPs affecting higher brain functions and chronic disease include a polymorphism in the 3'-untranslated region of the brain-expressed human Slit and Trk-like 1 (SLITRK1) gene, which strengthens an existing miR-189 target site, supports neurite growth and is involved in Tourette's syndrome and attention-deficit hyperactivity disorder. ${ }^{60}$ Also, college students homozygous for the ancestral A variant of an $A>G$ polymorphism, which changes miRNA-96 regulation of the aggressive behaviorassociated HTR1B gene, exhibit more conduct-disorder behaviors than individuals with the $\mathrm{G}$ allele. ${ }^{61}$ Our findings, in comparison implicate the ability to cope with miRNA dysregulation in brainrelated diseases. The genetic variations evident in the miRNA-608 gene itself (the 'seed' region) may alter its binding and thus activity. ${ }^{62}$ Consequently, in addition to the 3 '-untranslated region polymorphism, miRNA polymorphisms can potentially serve as additional biomarkers for PTSS, which warrants investigation.

There are several limitations to this study. The neurocircuitry models posit that in PTSD, the vmPFC fails to inhibit the amygdala, which leads to exaggerated threat responses and emotion regulation deficits. ${ }^{37}$ The currently observed effect thus reflects differential increases in the reactivity of the amygdala following prolonged exposure to stress and trauma. ${ }^{63}$ However, the genotype effect may also reflect pre-trauma vulnerability factors rather than consequences of stressful situations. ${ }^{8,29}$ The prospective studies examining the relation between the rs17228616 polymorphism and amygdala activity before and after stress insults of variable impact and length are required to disentangle this question. In addition, the currently studied population consisted of healthy Israel Defense Forces soldiers selected by extensive mental and physical screenings, and the rs17228616 polymorphism may have a different effect in the general population; genuine highly anxious populations should be tested before the results can be generalized. ${ }^{64}$ However, the use of a relatively healthy sample minimizes confounding factors such as comorbidities and medication use, thus representing a good approach for studying PTSS as a continuum. Nevertheless, the use of a small homogenous sample may have reduced PTSS variability, weakening the statistical power to detect all statistical effects, thus warranting additional studies on larger and more variable traumaprone populations, such as paramedics or firefighters. Further, the sample size of amygdala tissues and soldiers participating in the fMRI experiment may be too small to detect all the associations. It is also possible that the results of this study may be only applicable to chronic cases and not to acute stress symptoms.

To conclude, using a unique population at high risk for chronic stress exposure, we found that the rs17228616 gene variant influences neural responses to emotional stimuli; but that in the context of trauma load, its effect on PTSS is limited, plausibly due to intensified recruitment of the vmPFC. Importantly, our findings support the concept that noncoding RNAs may be involved in PTSS, and should be integrated into a neural model of the disorder. This study highlights the effectiveness of combined genomic and neuroimaging methods in the identification of intermediate phenotypes for PTSS and other psychiatric disorders and provides a further step towards personalized medicine in psychiatry by identifying previously neglected genetic variants of disease risks that may contribute to its vulnerability. Notably, our current findings point to one possible PTSS risk pathway, in which a risk genotype predisposes individuals to increased fear reactivity. This involves exaggerated amygdala responses that are supposedly downregulated by the vmPFC to avoid the development of PTSS. These findings also point to potential new treatment avenues by neuromodulation techniques targeting the amygdala or amygdala-vmPFC interactions.

\section{CONFLICT OF INTEREST}

The authors declare no conflict of interest.

\section{ACKNOWLEDGMENTS}

We are grateful to Dr Sebastian Kadener, Jerusalem, for fruitful discussions; and to the Netherland Brain Bank for the tissues and data. We acknowledge support by the European Research Council (Advanced Award 321501, to HS), the Legacy Heritage Science Initiative (LHSI) of the Israel Science Foundation (Grant no. 378/11, to HS), the Israel Center of Research Excellence for mass trauma (to HS), German Research Foundation Trilateral Cooperation Program (to HS) and by the US Department of Defense (award number W81XWH-11-2-0008, to TH), The Sagol network foundation (to TH), the Israel Centers of Research Excellence for cognitive studies (no. 51/11, to $\mathrm{TH}$ ), and the Israeli Ministry of Science, Technology and Space (Grant no. 3-11170, to TH.). SS-T was an incumbent of the Eshkol National postdoctoral fellowship.

\section{REFERENCES}

1 Lin T, Vaisvaser S, Fruchter E, Admon R, Wald I, Pine DS et al. A neurobehavioral account for individual differences in resilience to chronic military stress. Psychol Med 2015; 45: 1011-1023.

2 Taylor MK, Sausen KP, Potterat EG, Mujica-Parodi LR, Reis JP, Markham AE et al. Stressful military training: endocrine reactivity, performance, and psychological impact. Aviat Space Environ Med 2007; 78: 1143-1149.

3 Kessler RC, Sonnega A, Bromet E, Hughes M, Nelson CB. Posttraumatic stress disorder in the national comorbidity survey. Arch Gen Psychiatry 1995; 52: 1048-1060.

4 Cukor J, Wyka K, Jayasinghe N, Difede J. The nature and course of subthreshold PTSD. J Anxiety Disord 2010; 24: 918-923.

5 Grasso DJ, Cohen LH, Moser JS, Hajcak G, Foa EB, Simons RF. Seeing the silver lining: potential benefits of trauma exposure in college students. Anxiety Stress Coping 2012; 25: 117-136. 
6 Stein MB, Walker JR, Hazen AL, Forde DR. Full and partial posttraumatic stress disorder: findings from a community survey. Am J Psychiatry 1997; 154: 1114-1119.

7 Tull MT, Barrett HM, McMillan ES, Roemer L. A preliminary investigation of the relationship between emotion regulation difficulties and posttraumatic stress symptoms. Behav Ther 2007; 38: 303-313.

8 Admon R, Milad MR, Hendler T. A causal model of post-traumatic stress disorder: disentangling predisposed from acquired neural abnormalities. Trends Cogn Sci 2013; 17: 337-347.

9 Almli LM, Fani N, Smith AK, Ressler KJ. Genetic approaches to understanding posttraumatic stress disorder. Int J Neuropsychopharmacol 2014; 17: 355-370.

10 Schmidt U, Willmund G-D, Holsboer F, Wotjak CT, Gallinat J, Kowalski JT et al. Searching for non-genetic molecular and imaging PTSD risk and resilience markers: Systematic review of literature and design of the German Armed Forces PTSD biomarker study. Psychoneuroendocrinology 2015; 51: 444-458.

11 Lobo I, David IA, Figueira I, Campagnoli RR, Volchan E, Pereira MG et al. Brain reactivity to unpleasant stimuli is associated with severity of posttraumatic stress symptoms. Biol Psychol 2014; 103: 233-241.

12 Bogdan R, Pagliaccio D, Baranger DA, Hariri AR. Genetic moderation of stress effects on corticolimbic circuitry. Neuropsychopharmacology 2015; 41: 275-296.

13 Olsson A, Kross E, Nordberg SS, Weinberg A, Weber J, Schmer-Galunder S et al. Neural and genetic markers of vulnerability to post-traumatic stress symptoms among survivors of the World Trade Center attacks. Soc Cogn Affect Neurosci 2015; 10: $863-868$.

14 Poulton R, Andrews G, Millichamp J. Gene-environment interaction and the anxiety disorders. Eur Arch Psychiatry Clin Neurosci 2008; 258: 65-68.

15 Hanin G, Shenhar-Tsarfaty S, Yayon N, Yau YH, Bennett ER, Sklan EH et al. Competing targets of microRNA-608 affect anxiety and hypertension. Hum Mol Genet 2014; 23: 4569-4580.

16 Soreq $\mathrm{H}$. Checks and balances on cholinergic signaling in brain and body function. Trends Neurosci 2015; 38: 448-458.

17 Yehuda R, Seckl J, Minireview. Stress-related psychiatric disorders with low cortisol levels: a metabolic hypothesis. Endocrinology 2011; 152: 4496-4503.

18 Kaufer D, Friedman A, Seidman S, Soreq H. Acute stress facilitates long-lasting changes in cholinergic gene expression. Nature 1998; 393: 373-377.

19 Lettre G, Palmer CD, Young T, Ejebe KG, Allayee H, Benjamin EJ et al. Genomewide association study of coronary heart disease and its risk factors in 8,090 African Americans: the NHLBI CARe Project. PLoS Genet 2011; 7: e1001300.

20 Kibler JL, Joshi K, Ma M. Hypertension in relation to posttraumatic stress disorder and depression in the US National Comorbidity Survey. Behav Med 2009; 34: $125-132$.

21 Shin LM, Rauch SL, Pitman RK. Amygdala, medial prefrontal cortex, and hippocampal function in PTSD. Ann N Y Acad Sci 2006; 1071: 67-79.

22 Herringa RJ, Phillips ML, Fournier JC, Kronhaus DM, Germain A. Childhood and adult trauma both correlate with dorsal anterior cingulate activation to threat in combat veterans. Psychol Med 2013; 43: 1533-1542.

23 Mesulam M-M. Structure and function of cholinergic pathways in the cerebral cortex, limbic system, basal ganglia, and thalamus of the human brain. In: Bloom FE, Kupfer DJ (eds). Psychopharmacology: The Fourth Generation of Progress. Raven Press: New York, NY, USA, 1995, pp 135-146.

24 Meerson A, Cacheaux L, Goosens KA, Sapolsky RM, Soreq H, Kaufer D. Changes in brain microRNAs contribute to cholinergic stress reactions. J Mol Neurosci 2009; 40: 47-55.

25 Mineur YS, Obayemi A, Wigestrand MB, Fote GM, Calarco CA, Li AM et al. Cholinergic signaling in the hippocampus regulates social stress resilience and anxietyand depression-like behavior. Proc Natl Acad Sci USA 2013; 110: 3573-3578.

26 Tasker JG, Herman JP. Mechanisms of rapid glucocorticoid feedback inhibition of the hypothalamic-pituitary-adrenal axis. Stress 2011; 14: 398-406.

27 Pitman RK, Rasmusson AM, Koenen KC, Shin LM, Orr SP, Gilbertson MW et al. Biological studies of post-traumatic stress disorder. Nat Rev Neurosci 2012; 13: 769-787.

28 Etkin A, Wager TD. Functional neuroimaging of anxiety: a meta-analysis of emotional processing in PTSD, social anxiety disorder, and specific phobia. Am J Psychiatry 2007; 164: 1476-1488.

29 Admon R, Lubin G, Stern O, Rosenberg K, Sela L, Ben-Ami H et al. Human vulnerability to stress depends on amygdala's predisposition and hippocampal plasticity. Proc Natl Acad Sci USA 2009; 106: 14120-14125.

30 Mansvelder HD, Mertz M, Role LW. Nicotinic modulation of synaptic transmission and plasticity in cortico-limbic circuits. Semin Cell Dev Biol 2009; 20: 432-440.

31 Picciotto MR, Higley MJ, Mineur YS. Acetylcholine as a neuromodulator: cholinergic signaling shapes nervous system function and behavior. Neuron 2012; 76: 116-129.

32 Ben-Ari Y, Zigmond RE, Shute CCD, Lewis PR. Regional distribution of choline acetyltransferase and acetylcholinesterase within the amygdaloid complex and stria terminalis system. Brain Res 1977; 120: 435-445.
33 Meshorer E, Erb C, Gazit R, Pavlovsky L, Kaufer D, Friedman A et al. Alternative splicing and neuritic mRNA translocation under long-term neuronal hypersensitivity. Science 2002; 295: 508.

34 Shaltiel G, Hanan M, Wolf Y, Barbash S, Kovalev E, Shoham S et al. Hippocampal microRNA-132 mediates stress-inducible cognitive deficits through its acetylcholinesterase target. Brain Struct Funct 2013; 218: 59-72.

35 Milad MR, Wright Cl, Orr SP, Pitman RK, Quirk GJ, Rauch SL. Recall of fear extinction in humans activates the ventromedial prefrontal cortex and hippocampus in concert. Biol Psychiatry 2007; 62: 446-454.

36 Phelps EA, Delgado MR, Nearing KI, LeDoux JE. Extinction learning in humans: role of the amygdala and vmPFC. Neuron 2004; 43: 897-905.

37 Rauch SL, Shin LM, Phelps EA. Neurocircuitry models of posttraumatic stress disorder and extinction: human neuroimaging research-past, present, and future. Biol Psychiatry 2006; 60: 376-382.

38 Gomez-Merino D, Drogou C, Chennaoui M, Tiollier E, Mathieu J, Guezennec CY. Effects of combined stress during intense training on cellular immunity, hormones and respiratory infections. Neuroimmunomodulation 2005; 12 164-172.

39 Foa EB, Cashman L, Jaycox L, Perry K. The validation of a self-report measure of posttraumatic stress disorder: the posttraumatic diagnostic scale. Psychol Assess 1997; 9: 445.

40 Weathers FW, Litz BT, Herman DS, Huska JA, Keane TM. The PTSD checklist (PCL): reliability, validity, and diagnostic utility. Annual Meeting of the International Society for Traumatic Stress Studies, San Francisco, TX, USA, 1993.

41 Preacher KJ, Hayes AF. Asymptotic and resampling strategies for assessing and comparing indirect effects in multiple mediator models. Behav Res Methods 2008, 40: 879-891.

42 Alexander RP, Fang G, Rozowsky J, Snyder M, Gerstein MB. Annotating noncoding regions of the genome. Nat Rev Genet 2010; 11: 559-571.

43 Esteller M. Non-coding RNAs in human disease. Nat Rev Genet 2011; 12: 861-874.

44 Ghildiyal M, Zamore PD. Small silencing RNAs: an expanding universe. Nat Rev Genet 2009; 10: 94-108.

45 Barbash S, Shifman S, Soreq H. Global coevolution of human microRNAs and their target genes. Mol Biol Evol 2014; 31: 1237-1247.

46 Pirker S, Schwarzer C, Wieselthaler A, Sieghart W, Sperk G. GABAA receptors: immunocytochemical distribution of 13 subunits in the adult rat brain. Neuroscience 2000; 101: 815-850.

47 Green RC, Mesulam M-M. Acetylcholinesterase fiber staining in the human hippocampus and parahippocampal gyrus. J Comp Neurol 1988; 273: 488-499.

48 Nievergelt CM, Maihofer AX, Mustapic M, Yurgil KA, Schork NJ, Miller MW et al. Genomic predictors of combat stress vulnerability and resilience in U.S. Marines: a genome-wide association study across multiple ancestries implicates PRTFDC1 as a potential PTSD gene. Psychoneuroendocrinology 2015; 51: 459-471.

49 Ashley-Koch AE, Garrett ME, Gibson J, Liu Y, Dennis MF, Kimbrel NA et al. Genomewide association study of posttraumatic stress disorder in a cohort of IraqAfghanistan era veterans. J Affect Disord 2015; 184: 225-234.

50 Preacher KJ, Hayes AF. SPSS and SAS procedures for estimating indirect effects in simple mediation models. Behav Res Methods Instrum Comput 2004; 36 717-731.

51 Shin LM, Liberzon I. The neurocircuitry of fear, stress, and anxiety disorders. Neuropsychopharmacology 2009; 35: 169-191.

52 Haramati S, Navon I, Issler O, Ezra-Nevo G, Gil S, Zwang R et al. microRNA as repressors of stress-induced anxiety: the case of amygdalar miR-34. J Neurosci 2011; 31: 14191-14203.

53 Nadorp B, Soreq H. Predicted overlapping microRNA regulators of acetylcholine packaging and degradation in neuroinflammation-related disorders. Front $\mathrm{Mol}$ Neurosci 2014; 7: 9.

54 Zhang Y, Schiff D, Park D, Abounader R. MicroRNA-608 and microRNA-34a regulate chordoma malignancy by targeting EGFR, Bcl-xL and MET. PLoS ONE 2014; 9 : e91546.

55 Othman N, LLA In, Harikrishna JA, Hasima N. Bcl-xL silencing induces alterations in hsa-miR-608 expression and subsequent cell death in A549 and SK-LU1 human lung adenocarcinoma cells. PLoS One 2013; 8: e81735.

56 Akhtar N, Makki MS, Haqqi TM. MicroRNA-602 and microRNA-608 regulate sonic hedgehog expression via target sites in the coding region in human chondrocytes. Arthritis Rheumatol 2015; 67: 423-434.

57 Zimmerman G, Shaltiel G, Barbash S, Cohen J, Gasho CJ, Shenhar-Tsarfaty S et al. Post-traumatic anxiety associates with failure of the innate immune receptor TLR9 to evade the pro-inflammatory NFKB pathway. Transl Psychiatry 2012; 2: e78.

58 Mark GP, Rada PV, Shors TJ. Inescapable stress enhances extracellular acetylcholine in the rat hippocampus and prefrontal cortex but not the nucleus accumbens or amygdala. Neuroscience 1996; 74: 767-774.

59 Sanders SK, Shekhar A. Regulation of anxiety by GABAA receptors in the rat amygdala. Pharmacol Biochem Behav 1995; 52: 701-706. 
60 Abelson JF, Kwan KY, O'Roak BJ, Baek DY, Stillman AA, Morgan TM et al. Sequence variants in SLITRK1 are associated with Tourette's syndrome. Science 2005; 310: 317-320.

61 Jensen KP, Covault J, Conner TS, Tennen H, Kranzler HR, Furneaux HM. A common polymorphism in serotonin receptor 1B mRNA moderates regulation by miR-96 and associates with aggressive human behaviors. Mol Psychiatry 2008; 14: 381-389.

62 Zheng J, Deng J, Xiao M, Yang L, Zhang L, You Y et al. A sequence polymorphism in miR-608 predicts recurrence after radiotherapy for nasopharyngeal carcinoma. Cancer Res 2013; 73: 5151-5162.

63 van Wingen GA, Geuze E, Vermetten E, Fernández G. Perceived threat predicts the neural sequelae of combat stress. Mol Psychiatry 2011; 16: 664-671.
64 Shenhar-Tsarfaty S, Yayon N, Waiskopf N, Shapira I, Toker S, Zaltser D et al. Fear and C-reactive protein cosynergize annual pulse increases in healthy adults. Proc Natl Acad Sci USA 2015; 112: E467-E471.

\section{(c) (i)}

This work is licensed under a Creative Commons Attribution 4.0 International License. The images or other third party material in this article are included in the article's Creative Commons license, unless indicated otherwise in the credit line; if the material is not included under the Creative Commons license, users will need to obtain permission from the license holder to reproduce the material. To view a copy of this license, visit http://creativecommons.org/licenses/ by/4.0/

Supplementary Information accompanies the paper on the Translational Psychiatry website (http://www.nature.com/tp) 